\title{
Soft Robotic Glove with Motion Controlling System Based on BP Neural Network
}

\author{
Yi Fang \\ Nanjing Foreign Language School, Nanjing 210000, China. \\ hzhc_wjdi@163.com
}

Keywords: Mechanical glove, Leap motion controller, BP neural network.

\begin{abstract}
In order to facilitate the rehabilitation of patients with hand dyskinesia, a soft mechanical glove with the master-slave motion controlling system is proposed in this paper. Since the relationship between the input collected from movement of master hand and the output- the parameters needed to control slave glove is complex, the combination of the motion controlling system and BP neural network is applied in this paper to ensure the accuracy of simulating motion for slave glove. Firstly, the hardware and software design of the glove is demonstrated. And then, the training data of the fingertip-palm-distance and the corresponding rotating angles of steering to simulate the movement of master hand is calculated, based on which the BP neural network is established. Finally, the motion control error is proven within $5 \%$ by experimental validation. The BP neural network based controlling system demonstrated in this paper can also be applied in other similar problems in engineering practice.
\end{abstract}

\section{Introduction}

Nowadays, lots of patients suffer from hand dyskinesia caused by several common deceases like Parkinsonism or hemiplegic paralysis induced by cerebral apoplexy, which leads to the one-side-hand loss of ability of flexible motion or holding objects. Some biomimetic hand exotendon devices are proposed to aid the rehabilitation treatments by facilitating the disabled hand conduct repetitive practice.

Biomimetic hand exotendon devices used in these days mainly focus on exoskeleton structure, which are driven by directly stretching or discrete divers on knuckles [1,2]. Actuators of their driving system could be servo engine, air cylinder, pneumatic muscle, shape memory alloys etc. [3, 4]. Multi links and crank block are often used for transmission mechanism [5]. However, biomimetic hand exotendon devices normally have obvious drawbacks - large size and miscellaneous components. Additionally, the controlling systems, including open-loop control with pre-loaded data, closed-loop control with angle senor and pressure sensor, electromyographic signal control and active induction control based on visual reality technology [6], are either too simple to fulfill complicated tasks or too costly.

In this paper, a soft mechanical glove with the master-slave motion controlling system is proposed, the soft mechanical glove is utilized to improve the wearing comfort degree. Leap Motion is applied in getting master hand gesture data, the slave glove is driven by strings connecting fingers and servo engines to imitate the pose of master hand. Since the relationship between the input collected from movement of master hand and the output- the parameters needed to control slave glove is complex, the BP neural network is established to ensure the accuracy of simulating motion for slave glove. The BP neural network based controlling method provide a guideline for other similar problem in engineering practice.

\section{Composition of the Soft Robotic Glove}

Leap Motion is a three-dimensional data acquisition sensor based on computer vision technology, which is shown in Fig. 1. It can be used to identify static poses and motion states of hands, calculate the spatial coordinates of fingertips, joints, and palm direction vectors. In this paper, the Leap motion 
is used to construct the pose recognition module to obtain the space coordinates of master hand. According to the fingertip and palm space coordinates measured by the Leap Motion, the fingertippalm- distance Li can be calculated in the Microsoft Visual Studio software development platform and used as an evaluation index for the finger posture of master hand.

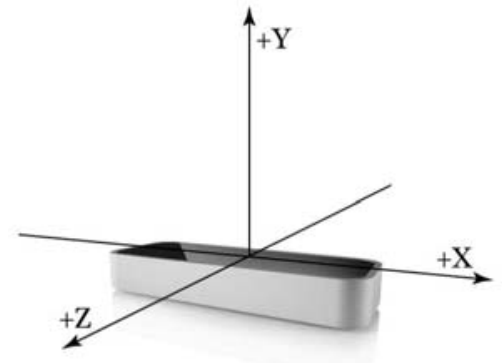

Figure 1. Leap Motion controller

In terms of slave hand side, the Arduino UNO development board is selected for main control module for its distinctive characteric of small size and high efficiency, the package information of Arduino UNO is shown in Fig. 2. Besides, due to the open source property of the hardware, there are abundant adaptable sensors and actuators to extend. According to the output datas of Microsoft Visual Studio, Arduino UNO main control module drive the steering gear rotate corresponding angle $\theta i$. The MG996 steering gear can meet the requirement of high torque and accurate rotation, with the steering rotating, the fingertip of slave hand is tracted by the strings which connects the joint of the soft glove on both sides of hand and the corresponding steering. The datas transmission between Microsoft Visual Studio and Arduino UNO development board is conducted by the serial port transmission module, and the LM2596 serves as the power supply module for this electronic hardware. The actuator module, serial communication module and power supply module are shown in Fig. 3. The mechnical soft robotic glove and corresponding controlling system is shown in Fig. 4.

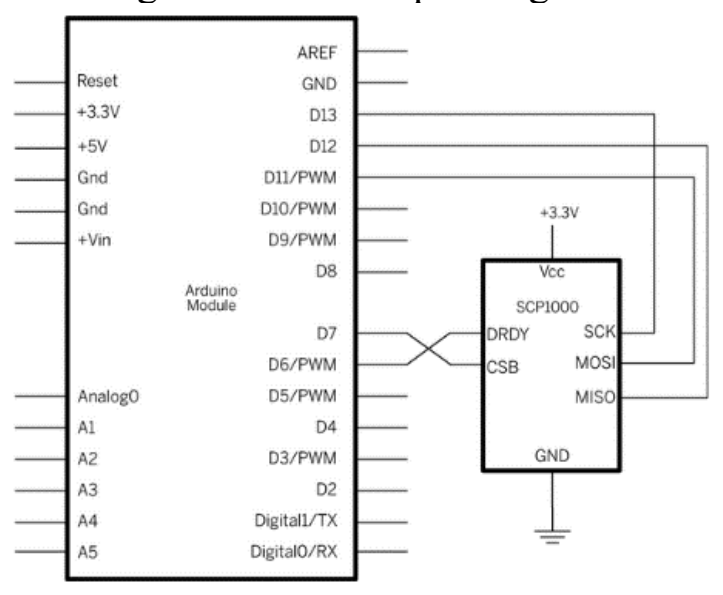

Figure 2. Arduino UNO package information

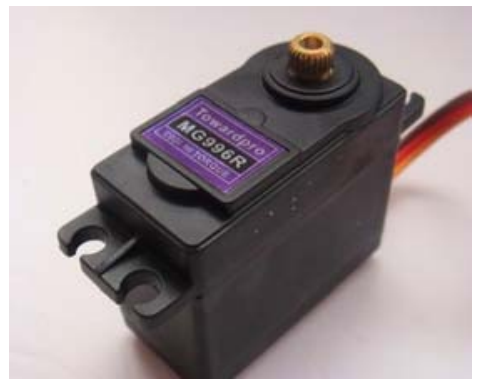

(a) MG996 steering gear

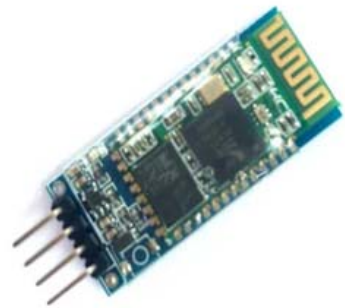

(b) Serial communication module $\overline{\text { (c) Power supply module }}$ Figure 3. Electronic modules

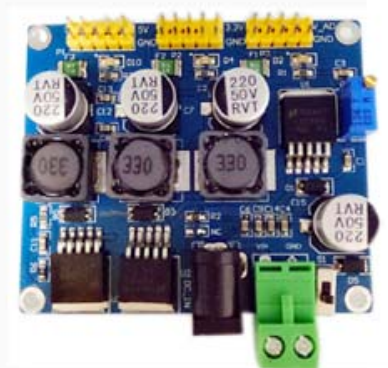




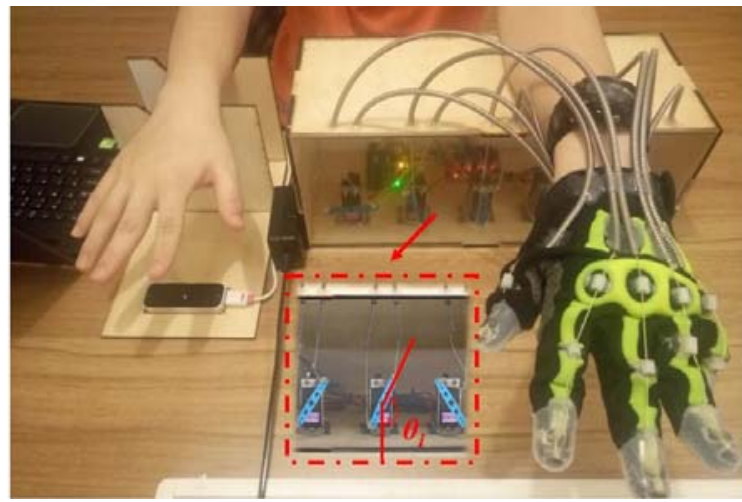

Figure 4. Mechnical soft robotic glove

However, during the data processing procedure in Visual Studio, since the relationship between the input- the fingertip- palm- distance $\mathrm{Li}$ and the output- the rotating angle $\theta \mathrm{i}$ needed to drive slave hand to imitate the pose of master hand is nonliner, it is difficult to describe the maping using mathematical formula. In this paper, the BP neural network controlling model is established to ensure the accuracy of imitating motion for slave glove.

\section{BP Neural Network Based Controlling Method}

\subsection{Principle of BP Neural Network.}

BP neural network uses Error Back Propagation Algorithm (BP algorithm) to correct the hidden layer weights along the negative gradient of the error function in order to make the mean square error between the actual output and the expected output of the network minimize, make the multi-layer feed-forward network learn and optimize continuously according to the sample, reach the effect that approaches any nonlinear function with arbitrary precision.

According to the system sample set (X, Y), the number of nodes in network input layer $\mathrm{n}$, the number of hidden layer nodes 1 , the number of output layer nodes $\mathrm{m}$, the connection weights of initialized layers and the hidden layer threshold $a$ and the output layer threshold $b$ are determined. For the input variable xi, calculate the hidden layer output $\mathrm{Hj}$ :

$$
H_{j}=f\left(\sum_{i=1}^{n} \omega_{i j} x_{i}-a_{j}\right), \quad j=1,2, \ldots, l
$$

Where the hidden layer excitation function $\mathrm{f}$ can be expressed into hyperbolic tangent function:

$$
f(x)=\frac{2}{1+e^{-2 x}}-1
$$

According to the hidden layer output $\mathrm{Hj}$, connection weight and threshold $\mathrm{Bk}$, the output $\mathrm{Ok}$ can be predicted by BP neural network:

$$
O_{k}=\sum_{j=1}^{l} H_{j} \omega_{j k}-b_{k}, \quad k=1,2, \ldots, m
$$

According to neural network prediction output Ok and expected output YK, the error eke can be calculated:

$$
e_{k}=Y_{k}-O_{k}
$$

According to the network prediction error ek update the network connection weights iteratively.

$$
\begin{gathered}
\omega_{i j}{ }^{s+1}=\omega_{i j}{ }^{s}+\eta\left(1-H_{j}\right) x(i) \sum_{k=1}^{m} \omega_{j k} e_{k}, \quad i=1,2, \ldots, n ; j=1,2, \ldots, l \\
\omega_{j k}{ }^{s+1}=\omega_{j k}{ }^{s+1}+\eta H_{j} e_{k}, \quad j=1,2, \ldots, l ; k=1,2, \ldots, m
\end{gathered}
$$

Where, $\eta$ is the learning rate, the network node threshold am, $\mathrm{Bk}$ is updated according to the network prediction error ek.

$$
a_{j}^{s+1}=a_{j}^{s+1}+\eta H_{j}\left(1-H_{j}\right) \sum_{k=1}^{m} \omega_{j k} e_{k}, \quad j=1,2, \ldots, l
$$




$$
b_{k}^{s+1}=b_{k}^{s}+e_{k}, \quad k=1,2, \ldots m
$$

The iterative process continues until the calculation error reaches the expected value.

\subsection{Modeling.}

In order to decribe the relationship between fingertip - palm- distances Li and rotating angles of steering $\theta \mathrm{i}$, and realize accurate master-slave control, the BP neural network is established through data training. Ten sets of sample data were obtained by sampling measurment, as shown in Table 1 . Since the trajectory of each fingertip is different and independent, and the high-dimensional BP neural network requires a large amounts of sampling points, the single-input-single-output BP neural network model is constructed for each finger.

Table 1. Sampling points of fingertip - palm- distances and corresponding rotating angles

\begin{tabular}{cccccccccccc}
\hline \multirow{2}{*}{ Numble } & \multicolumn{3}{c}{ Thumb } & \multicolumn{3}{c}{ Index finger } & \multicolumn{3}{c}{ Middle finger } & \multicolumn{3}{c}{ Ring finger } & \multicolumn{3}{c}{ Little finger } \\
\cline { 2 - 12 } & $L_{T}$ & $\theta_{T}$ & $L_{I}$ & \multicolumn{2}{c}{$\theta_{I}$} & $L_{M}$ & $\theta_{M}$ & $L_{R}$ & $\theta_{R}$ & $L_{L}$ & $\theta_{L}$ \\
\hline Case 1 & 40.5 & 18.4 & 63.1 & 15.2 & 62.0 & 24.9 & 69.9 & 11.3 & 51.1 & 1.2 \\
Case 2 & 44.2 & 25.9 & 68.9 & 27.2 & 66.7 & 33.2 & 71.5 & 14.0 & 56.3 & 3.2 \\
Case 3 & 49.7 & 41.0 & 74.4 & 44.1 & 73.0 & 47.4 & 76.4 & 25.3 & 61.5 & 7.7 \\
Case 4 & 54.1 & 56.9 & 77.1 & 54.0 & 77.4 & 59.3 & 82.4 & 47.3 & 65.1 & 13.2 \\
Case 5 & 57.4 & 70.4 & 83.3 & 82.2 & 83.9 & 79.9 & 84.6 & 57.5 & 72.2 & 33.5 \\
Case 6 & 63.1 & 97.7 & 87.7 & 104.7 & 90.7 & 104.1 & 90.3 & 89.4 & 76.3 & 52.2 \\
Case 7 & 65.4 & 109.2 & 91.6 & 125.2 & 95.9 & 123.3 & 95.4 & 121.7 & 79.8 & 72.1 \\
Case 8 & 69.9 & 132.2 & 94.1 & 138.2 & 99.7 & 137.6 & 99.5 & 146.6 & 85.9 & 114.2 \\
Case 9 & 76.6 & 162.7 & 99.7 & 162.6 & 106.5 & 160.7 & 102.1 & 159.7 & 91.9 & 156.0 \\
Case 10 & 80.9 & 177.2 & 105.7 & 178.9 & 111.1 & 174.0 & 107.3 & 178.3 & 96.3 & 180.0 \\
\hline
\end{tabular}

The MATLAB neural network toolbox is envoked in the Microsoft Visual Studio software development platform for BP neural network data training. The network contains two hidden layers, with the number of nodes is 40 and 60 respectively. The hyperbolic tangent sigmoid function is used as excitation function. The learning rate $\eta$ is taken as 0.01 , the training error threshold is $1 \mathrm{e}-6$, and the training mean squared error convergence curve of each finger is shown in Fig. 5.

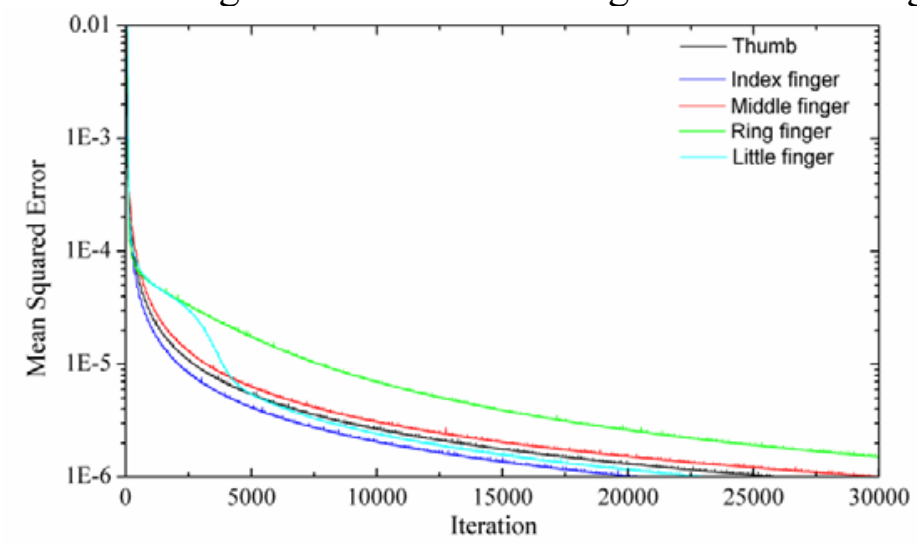

Figure 5. Mean square error convergence curve of BP neural network data training process

\subsection{Validation.}

In order to verify the prediction accuracy of the BP neural network model between the fingertippalm- distance and the rotating angle of the steering, take the index finger for example, the angle $\theta 1$ denotes the accurate required rotating angle of the steering for realizing the master-slave controlling mode, and the rotation angle $\theta 2$ denotes the prediction result of the BP neural network. The prediction error can be expressed by the ratio of the angle difference $\delta \theta$ and the required angle $\theta 1$, the results are shown in Table 2.

Table 2. Comparison of prediction angle and accurate result

\begin{tabular}{ccccc}
\hline $\begin{array}{c}\text { Validation } \\
\text { gourp }\end{array}$ & $\begin{array}{c}\text { Fingertip-palm- } \\
\text { distance } L_{I}(\mathrm{~mm})\end{array}$ & $\begin{array}{c}\text { Accurate value } \\
\theta_{1}\left({ }^{\circ}\right)\end{array}$ & $\begin{array}{c}\text { Perdiction value } \\
\theta_{2}\left({ }^{\circ}\right)\end{array}$ & $\begin{array}{c}\text { Error } \\
\delta \theta / \theta_{1}(\%)\end{array}$ \\
\hline v1 & 68.2 & 25.4 & 24.7 & 2.88 \\
v2 & 83.5 & 82.2 & 86.7 & 4.13 \\
v3 & 98.8 & 159.2 & 165.8 & 4.17 \\
\hline
\end{tabular}


As is seen from Table 2, the calculation error between the predicted result and the accurate value is within 5\%, which means the BP neural network controlling model established through data training can reach sufficient accuracy.

\section{Summary}

In this work, a soft mechanical glove with the master-slave motion controlling system is proposed, the hardware and software design of the glove is demonstrated in detail: Leap Motion is applied in getting the fingertip- palm- distance of master hand, the mechnical glove of slave side is driven by strings connecting fingers and servo engines to imitate the pose of master hand. In order to simulate the nonliner relationship between the fingertip- palm- distance and rotating angle of steering, the BP neural network is established by invoking the MATLAB BP neural network toolbox in Visual Studio, data training process is conducted based on the sampling measurment results. According to the validation of calculation, the prediction error of established BP neural network is within 5\%. Thus, it is anticipated that The BP neural network based controlling system demonstrated in this paper can also be applied in other similar problems in engineering practice.

\section{References}

[1]. Li H, Xin L, Wei S, et al. Research Status of Rehabilitation Robot for Hand Function [J]. Machine Design \& Research, 2014.

[2]. Cheri A, Giovacchini F, Vitiello N, et al. HANDEXOS: towards an exoskeleton device for the rehabilitation of the hand[C]// IEEE/RSJ International Conference on Intelligent Robots and Systems. IEEE Press, 2009:1106-1111.

[3]. Kim Y M, Jung S Y, Moon I. Design of a wearable upper-limb rehabilitation robot using parallel mechanism[C]// ICCAS-SICE. IEEE, 2009:785-789.

[4]. Bee J H, Kim Y M, Moon I. Wearable hand rehabilitation robot capable of hand function assistance in stroke survivors[C]//Biomedical Robotics and Bio mechatronics (Bio Rob), 2012 4th IEEE RAS \& EMBS International Conference on. IEEE, 2012: 1482-1487.

[5]. Ito S, Kawasaki H, Disfigure Y, et al. A design of fine motion assist equipment for disabled hand in robotic rehabilitation system [J]. Journal of the Franklin Institute, 2011, 348(1): 79-89.

[6]. Xu G Z, Song a G, Li H J. System design and control technique of robot-aided rehabilitation [J]. Journal of Clinical Rehabilitative Tissue Engineering Research, 2009. 